\title{
Design of the Commutator for the Cylindrical Type Magnetic Coupling Capping Head
}

\author{
LIU Bin \\ School of Mechanical Engineering \\ Nanjing Institute of Industry Technology \\ Nanjing, Jiangsu, China \\ liub@niit.edu.cn
}

\author{
GONG Xiaoqun \\ School of Mechanical Engineering \\ Nanjing Institute of Industry Technology \\ Nanjing, Jiangsu, China \\ gongxq@niit.edu.cn
}

\begin{abstract}
A kind of commutator for the cylindrical type magnetic coupling capping head is designed for capping machine. According to the performance requirements of the torque from the capping head, the designed cylindrical structure magnetic coupling, torque can be adjusted linearly, the magnetic coupling torque is calculated and the performance experiment for the capping head is carried out. The result shows that the designed commutator for the cylindrical type magnetic coupling capping head meets the demand for the main parts. The overall performance of the commutator for the cylindrical type magnetic coupling capping head is valuable for engineering applications.
\end{abstract}

Keywords-capping head; cylindrical type magnetic coupler torque; performance; commutator

\section{INTRODUCTION}

The cylindrical type magnetic coupling capping head is widely used in bottle filling production such as beer filling production, cola drinks filling production and some gas juice beverage filling production. This type of filler is perhaps the most widely used machine in small bottle filling operations. With the increasing speed of the bottle filling line $(24,000$ bottles per hour or more), the productivity of the capping mahine for the cylindrical type magnetic coupling capping

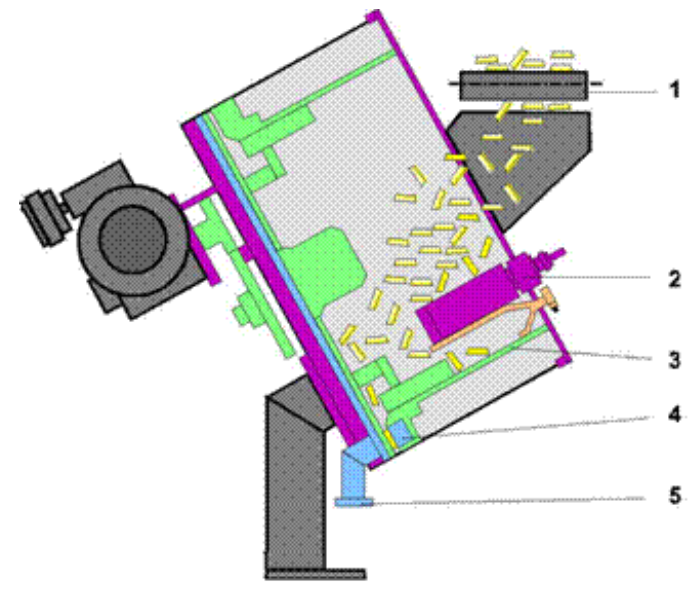

Fig.1 Capping machine for beverage filling production

Sponsored by the open foundation on Precision manufacturing engineering and technology research of Jiangsu development center (ZK13-01-01). head, particularly the performance requirements of the cylindrical type magnetic coupling capping head are increasing. Improving the efficency of the cap-orientation method of the cylindrical type magnetic coupling capping head is the key to meet the speed of bottle filling line, so it is necessary to design a high efficency commutator for the cylindrical type magnetic coupling capping head.

According to the principle, the cap-orientation method of the cylindrical type magnetic coupling capping head can be divided into negative cap-orientation method and positive caporientation method. The negative cap-orientation method of the cylindrical type magnetic coupling capping includes mechanical stirring cap-orientation method, electromagnetic vibration cap-orientation method and magnetic rotary caporientation method $^{[1]}$. In all above-mentioned cap-orientation methods, the caps in the negative direction must be eliminated in the course of production, i.e. in the course of production, a special constructions are designed to eliminate the caps in the negative direction. All the caps eliminated in the negative direction go back to the stock bin, so the number of the caps export to the capping-machine decreases dramatically, in theory, the number of the caps in the negative direction is $50 \%$ of the total caps. So, the production of each capping machine by eliminating the caps in the negative direction with the negative cap-orientation method is less than 10000 bottles per hour. The speed of cap feeding rate directly affects and limits the production efficiency of the capping machine. The capping machine is shown in the fig.1.

At present, the positive cap-orientation equiment of the cylindrical type magnetic coupling capping head at home and aboard, usually arrange the cylindrical type magnetic coupling capping head by magnetic capping stock bin. The arranged cylindrical type magnetic coupling capping heads are put in positive and negative direction, then, the cylindrical type magnetic coupling capping heads in the negative direction are reversed in the course of feeding, finally, all of the cylindrical type magnetic coupling capping heads lie in the positive direction and are transported to the slideway to supply the capping machine[2,3].

In the paper, a positive cap-orientation method for the cylindrical type magnetic coupling capping head is supposed. The process and the design of the cylindrical type magnetic coupling capping based on the positive cap-orientation method 
are introduced. Based on the position cap-orientation method, a commutator is designed and machined, by test, the production efficiency of the capping machine with the designed commutator is shown.

\section{STRUCTURE OF THE COMMUTATOR}

In the paper, the structure of commutator for the cylindrical type magnetic coupling capping head is shown in the fig.2.

The cylindrical type magnetic coupling capping heads in positive direction and negative direction are arranged by the specific caping-orientation machine of the commutator in right direction, and the cylindrical type magnetic coupling capping heads in the right direction are supplied to the capping machine. The cap-orientation method of the commutator is the postive cap-orientation, the accuracy rate of the caporientation of the commutator is $100 \%$. In the commutator, the cylindrical type magnetic coupling capping heads in the negative direction are not required to be aliminated, on the contrary, the cylindrical type magnetic coupling capping heads in the negative direction are reversed, so the efficiency of the commutator is twice as high as the efficiency of the commutator based on the negative cap-orientation method.

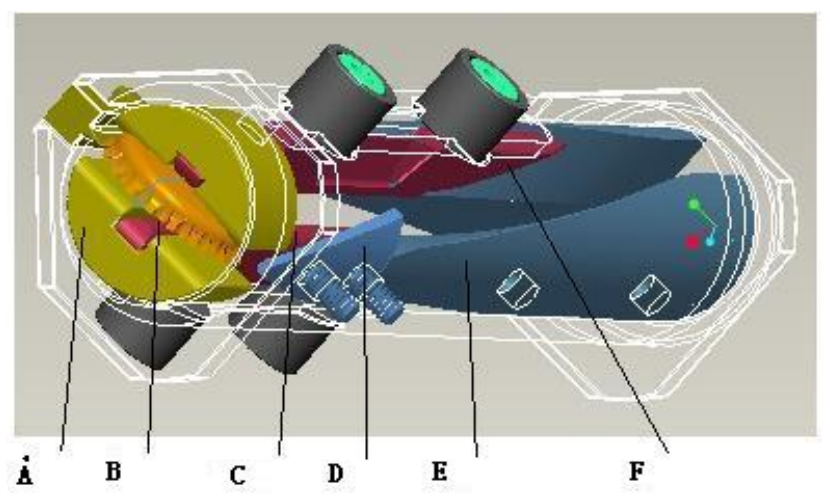

Fig. 2. The structure of commutator

As show in the fig.2, $\mathrm{A}$ in the picture is represented as the cylinder of the commutator, $\mathrm{B}$ in the picture is represented as the edge guide plate, $\mathrm{C}$ in the picture is represented as the vibration node, $\mathrm{D}$ in the picture is represented as the small wing tip type guide plate, $\mathrm{E}$ in the picture is represented as the long wing tip type guide plate, $\mathrm{F}$ in the picture is represented as the short wing tip type guide plate.

\section{StRUCTURE DESIGN OF THE COMMUTATOR}

\section{A. The Process of the Cylindrical Type Magnetic Coupling Capping Heads Changes Direction in the Commutator}

The commutator for the cylindrical type magnetic coupling capping heads is used to be placed vertically, after the cylindrical type magnetic coupling capping heads entering the entrance of the commutator from slideway, the cylindrical type magnetic coupling capping heads are guided to orientate by edge guide plate. the cylindrical type magnetic coupling capping heads are orientated through the characteristic structure of the edge of the cylindrical type magnetic coupling capping heads in the entrance of edge guide plate. Fig. 3 shows the characteristic structure of the entrance of the commutator.

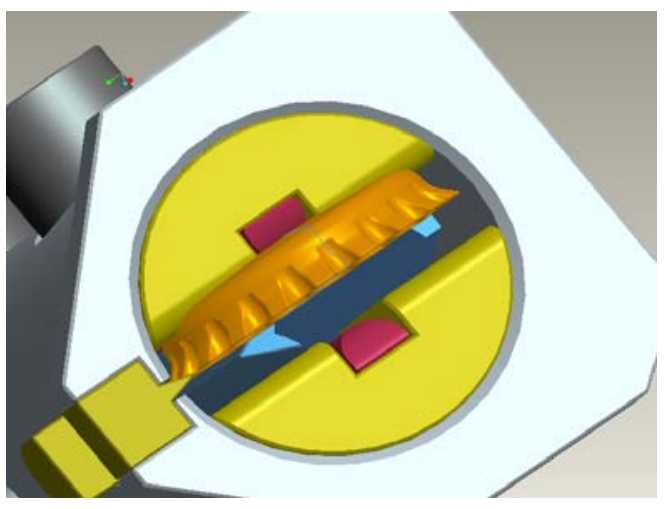

Fig. 3. Structure of the entrance of commutator

The cylindrical type magnetic coupling capping heads slide downward along the edge guide plake after entering the commutator, because the slideway is straight, the cylindrical type magnetic coupling capping heads can't rotate in the course of sliding downward. After sliding into the tip of the small wing tip type guide plate linking with the edge of the commutator, the cylindrical type magnetic coupling capping heads enter the spiral slide way, whiletime, the edge of the cylindrical type magnetic coupling capping heads are restricted by the edge of the small wing tip type guide plate, and the cap parts of the cylindrical type magnetic coupling capping heads are restricted by the spiral curved surface of the small wing tip type guide plate, so the cylindrical type magnetic coupling capping heads fall and rotate. This process is shown in fig. 4 .

As shown in fig.4, before breaking away from the small wing tip type guide plate and the short wing tip type guide plate, the cylindrical type magnetic coupling capping heads are guided by the long wing tip type guide plate, and the dropping rotation attitude of the cylindrical type magnetic coupling capping heads is decided by the long wing tip type guide plate, and the short wing tip type guide plate guides the edge and the cap parts of the cylindrical type magnetic coupling capping heads.

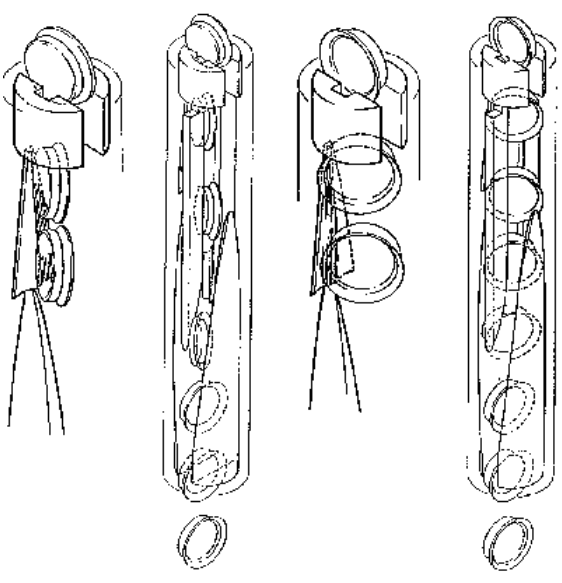

Fig.4. The process of rotation and drop of the cylindrical type magnetic coupling capping heads 
In the course of rotating and dropping of the cylindrical type magnetic coupling capping heads, on the one hand, the vibration rod as shown in the fig. 5 acts as the assistant guide on the dropping attitude of the cylindrical type magnetic coupling capping heads, on the other hand, the vibration rod vibrates at a certain frequency under outside force to prevent the cylindrical type magnetic coupling capping heads from blocking or retention in the commutator.[4,5]

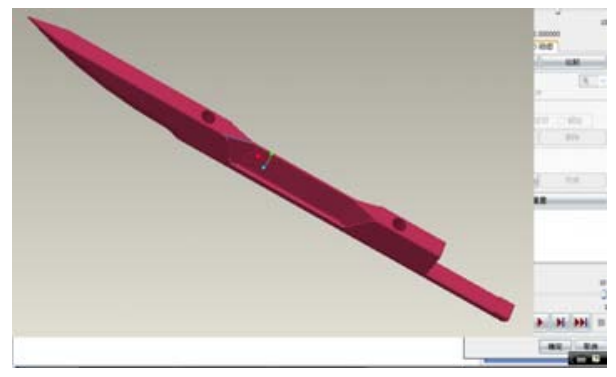

Fig.5. The structure of vibration node

\section{B. Determination and calculation of the size of the main parts} of the commutator

According to the national standard GB / T 13521-1992, the external diameter of the cylindrical type magnetic coupling capping heads is $\Phi \mathrm{D}=32.1 \pm 0.02 \mathrm{~mm}$, the height is $\mathrm{H}=6.75_{0}^{+0.15}$ (for the standard cylindrical type magnetic coupling capping heads). considering the clearance, the size of the entrance is designed as $\mathrm{H}_{1}=7.8 \mathrm{~mm}$, and the diameter $\Phi D_{1}=33 \pm 0.2 \mathrm{~mm}$.

According to the design requirements, in the course of dropping, the cylindrical type magnetic coupling capping heads in positive direction and in the negative direction must rotate 90 degree clockwise or counterclockwise. In the course of dropping, the cylindrical type magnetic coupling capping heads fall $2 \mathrm{~mm}$ and rotate 1 degrees in the meanwhile, so the total lead of the spiral slide way is:

$$
L=2 \times 90=180 \mathrm{~mm}
$$

The first section of the small wing tip type guide plate rotates 30 degrees, so the lead of the small wing tip type guide plate is:

$$
L=2 \times 30=60 \mathrm{~mm}
$$

Including $30 \mathrm{~mm}$ guide distance of the entrance of the commutator and $20 \mathrm{~mm}$ guide distance of the exit of the commutator, the total length of the commutator is $230 \mathrm{~mm}$.

\section{THE DESIGN OF THE KEY PARTS OF THE COMMUTATOR SURFACE MODELING}

The key parts of the cylindrical type magnetic coupling capping heads have special space surface, such as the small wing tip type guide plate, long wing tip type guide plate and short wing tip type guide plate. the space surface of the several parts can't be decribed by specific mathematical expression. The several parts are designed mainly based on the movement locus in the course of dropping of the standard cylindrical type magnetic coupling capping heads. The movement locus is the key to design the long wing tip type guide plate, the small wing tip type guide plate and the short wing tip type guide plate.
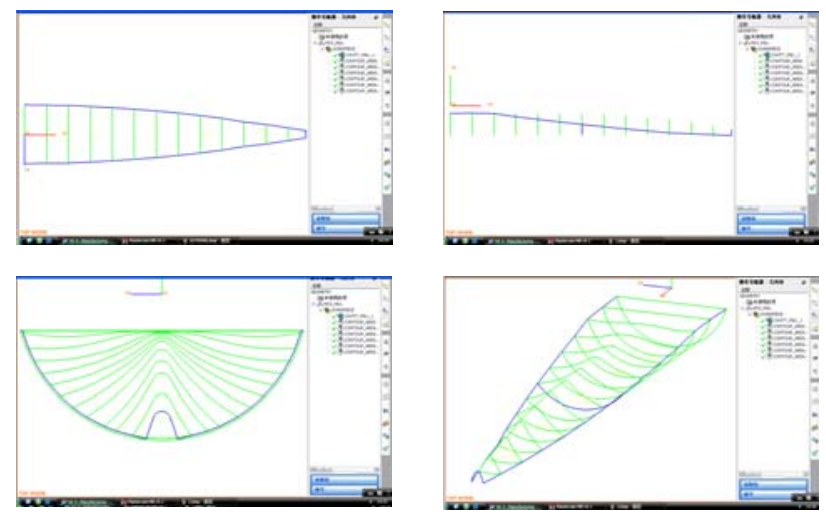

Fig.6. The design of the long wing tip type guide plate

Taking the long wing tip type guide plate of the commutator for example, the three dimension surface design of the several parts of the commutator is illustrated.

For the design of the long wing tip type guide plate, firstly, the reference line is determined initially, then, the reference line is divided by the isometric cross section. Based on the above mentioned size, the long wing tip type guide plate is designed by the UG software as shown in fig. 6 .

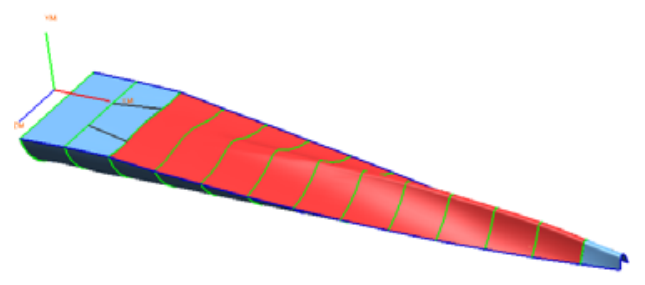

Fig.7. the processing technic of the long wing tip type guide plate of the commutator

In the four graphs above mentioned as shown in the fig.6, the Machining trajectory of the long wing tip type guide plate of the commutator is designed by the UG software. From four different directions, the machining trajectory of the long wing tip type guide plate of the commutator is shown in fig.6. As shown in the fig.7, the long wing tip type guide plate of the commutator is machined by iso -height plane rough machining firstly, then, the long wing tip type guide plate of the commutator is machined by surface finishing[6].
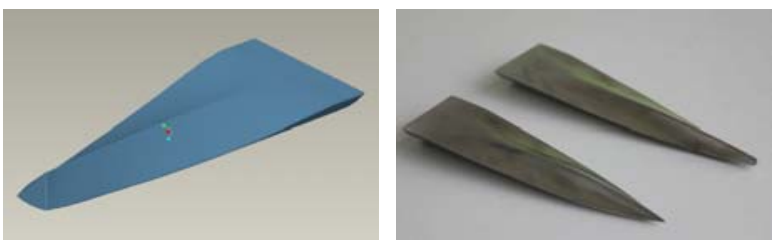

Fig. 8. Picture of real products 
The real products of the long wing tip type guide plate are produced based on the above mentioned design, the real products of the long wing tip type guide plate are shown in fig.8.

\section{CONCLUSION}

Through the experiment, the overall structure scheme of the commutator for the cylindrical type magnetic coupling capping heads is proved to be correct, and the design of structure size of the commutator is reasonable.

Through the test for the commutator, the results show that the commutator operates stably, the production capacity can reach to 36000 bottle per hour to meet the needs of bottle capping product line of the beer, beverage.

The volume of the commutator for the cylindrical type magnetic coupling capping heads is small in size, and the length of reversing zone of commutator is short. The efficiency of the commutator can reach $100 \%$, the supply speed and the output of the commutator are high. In the product process of capping machine for the cylindrical type magnetic coupling capping heads, in the paper, we have solve the key problem affecting the production rate of capping machine. The design of the commutator deserves popularizing and applying in engineerings.

\section{Acknowledgment}

The paper is supposed by Supposed by the open foundation on Precision manufacturing engineering and technology research of Jiangsu development center (ZK13-0101)

\section{References}

[1] Z. K. Zhao, "The design pringiple and key point of magnetic force turning order equipment for crown cap,” Packing and Food Machinery, vol. 20, no. 4, pp. 7-10, 2002.

[2] Z. X. Zhou, "Calculation and application of the transmission torque of magnetic coupling,” Fluid Machinery, vol. 21, no.11, pp. 43-47, 1993.

[3] H. Yang, H. Zhao, "Finite element analysis of the torque characteristics of the fast electromagnetic clutch,” Light Industry Machinery, no. 1, pp. 74-76, 2004.

[4] J. R. Liu, "Design of magnetic coupling transmission of chemical pump,” Fluid Machinery, vol.29, no. 12, pp. 36-37, 2001.

[5] L.Q. Li, X. X. Chen, J. Q. Wang, "application of magnetic drive structure in screwed rotating cover,” Light Industry Machinery, vol. 31, no. 4, pp. 18-21,2013.

[6] B. Liu, X. Q. Gong, “Cylindrical type magnetic coupling rotary capping heads,” Light Industry Machinery, vol. 29, no. 6, pp. 1-3 ,2011. 